\title{
The value of ultrasound diagnosis in the multidisciplinary approach of cutaneous tumours. Case report
}

\author{
Avram Manea1, Diana Crisan², Alexandru Florin Badea ${ }^{3}$, Dinu Iuliu Dumitrascu ${ }^{3}$, \\ Mihaela Baciut ${ }^{4}$, Simion Bran ${ }^{4}$, Ileana Mitre ${ }^{1}$, Maria Crisan ${ }^{5}$, Grigore Baciut ${ }^{1}$
}

${ }^{1}$ Cranio-Maxillofacial Surgery and Radiology Department, "Iuliu Hatieganu” University of Medicine and Pharmacy, Cluj-Napoca, Romania, ${ }^{2}$ Dermatology and Allergic Diseases Clinic, University Clinic Ulm, Germany, ${ }^{3}$ Anatomy and Embriology Department, "Iuliu Hatieganu" University of Medicine and Pharmacy, Cluj-Napoca, ${ }^{4}$ Maxillofacial Surgery and Implantology Department, "Iuliu Haţieganu" University of Medicine and Pharmacy, Cluj-Napoca, Romania, ${ }^{5}$ Dermatology and Venereology Department, University of Medicine and Pharmacy "Iuliu Hatieganu" Cluj-Napoca, Romania

\begin{abstract}
We report the case of a senior patient, presenting with two cutaneous tumours in which the clinical and dermoscopic examination pointed towards a basal cell carcinoma. Ultrasound revealed highly vascularised tumours and elastography showed increased rigidity. Histological examination of both lesions following complete resection was consistent with squamous cell carcinomas. The distinctive feature of this case consists of the complex non-invasive diagnostic imaging of the tumour morphology, performed in real time, revealing aspects compatible with highly proliferative malignant tumours. This case emphasises the importance of ultrasound diagnosis in a multi-disciplinary approach for better and more predictable results.
\end{abstract}

Keywords: basal cell carcinoma; squamous cell carcinoma; dermoscopy; skin neoplasms; ultrasonography

\section{Introduction}

Skin cancer is the most frequent form of human cancer $(9-13 \%$ of all diagnosed forms of cancer); $20 \%$ of people over the age of 70 had at least one basal cell carcinoma (BCC) or spinocellular carcinoma (SCC) [1].

BCCs develop slowly in photo-exposed areas (being directly linked to UV-exposure) or in mechanically stressed areas. The tumours present as nodules, papules or patches, which gradually increase in surface and often ulcerate. Typical clinical signs include prominent telangiectasias on the surface and a pearly white translucent border. Dermatoscopy may be used for more accurate di-

Received 30.04.2017 Accepted 19.06.2017

Med Ultrason

2018, Vol. 20, No 1, 108-110

Corresponding author: Maria Crisan

Dermatology and Venereology Department, University of Medicine and Pharmacy

"Iuliu Hatieganu" Cluj-Napoca

3-5 Clinicilor street, 400006, Cluj-Napoca,

Romania

Phone: +40744766496

E-mail: maria.crisan@umfcluj.ro agnosis (arborising "treelike" telangiectasia, ulcerations, etc.) [2].

SCCs are more aggressive tumours, have metastatic capacity and occur especially in elderly patients. Two categories can be distinguished: aggressive, highly metastatic SCC developing on irradiated areas and chronic ulcerations, and SCC with low aggressiveness and lower metastatic risk, developing on photo-aggressed areas. Photo-induced SCCs develop more frequently on the forehead, scalp, ears, the back of the hand, often from precursor actinic keratosis lesions. Initially in the form of thick adherent scales, the lesions develop during a relatively short period of time into firm tumorous masses with well-defined edges. In the maxillo-facial area, the most common regional metastatic site appears to be the parotid gland [3-5].

The diagnosis of suspect non-pigmented cutaneous tumours is usually clinical. Post-excisional histology confirms the diagnosis. Ultrasonography (US) provides valuable real time information for differential diagnosis and therapeutic approach such as lateral extension and depth, vascularisation, rigidity. Elastography is a non-in- 
vasive technique which determines the elasticity of soft tissues. Reduced elasticity correlates with tumour congestion and hypervascularization [6-8].

This case report emphasizes the importance of US in evaluating cutaneous tumours and its impact on therapeutic approach optimisation.

\section{Case report}

A 83 year-old patient presented in Dermatology Clinic (Outpatient Unit) with two nodular and irregularly shaped lesions of the skin, situated on the left and right infraorbital region (fig 1a). The lesions were protruding, erythematous, with an irregular surface and relatively well defined edges, with areas of ulcerations and hemorrhagic crusts. Both tumours initially started as small asymptomatic papules, which developed over several months to indolent bleeding nodules. The left tumour was measuring $2 / 1 \mathrm{~cm}$ and was situated at $1.5 \mathrm{~cm}$ inferior to the infraorbital margin. The right tumour was situated at $1 \mathrm{~cm}$ inferior to the infraorbital margin, measuring $0.5 / 1.5 \mathrm{~cm}$.

No lymphnodes were palpable in the head and neck region. Also, she did not recall any local trauma in the

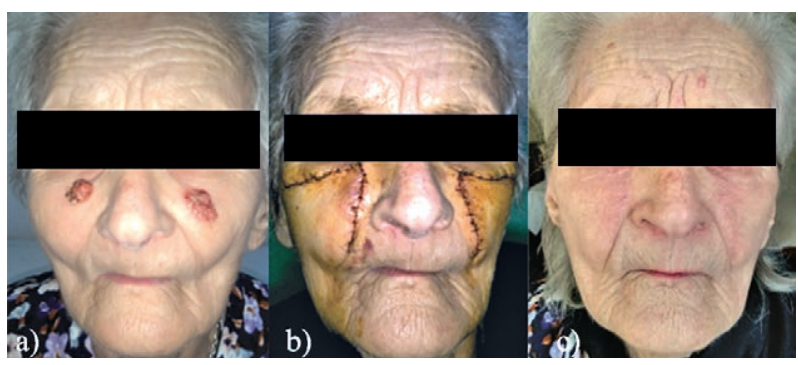

Fig 1. Clinical aspect showing: a) nodular, ulcerated, symmetrical tumours - the patient's first visit; b) 7 days after surgery; c) 5 months after surgery. past. Besides belonging to phototype II, she worked as a courier for more than 40 years, which implied outdoor activities. Dermatoscopy (fig 2a) of both lesions showed numerous dilated asymmetrical arborising vessels on the surface of both lesions. Bilateral BCC was suspected.

Gray scale US (fig $2 b$ ) revealed two well defined, hypoechogenic, inhomogeneous, ulcerated tumours $(15 / 4$ $\mathrm{mm}$ in the right side and $20 / 3.2 \mathrm{~mm}$ in the left side) located in the dermis and imprinting the hypodermis. Doppler US (fig 2c) found peripheral and central tumour hypervascularization with numerous dilated vessels, and 3, respectively 4 vascular pedicles. Qualitative elastography (fig $2 \mathrm{~d}$ ) revealed an increased rigidity of both tumours. Imagistic aspects, especially the presence of abundant basal vascularisation, raised the suspicion of achromic melanoma or SCC.

Surgical excision of the tumours was performed in general anesthesia (fig $1 \mathrm{~b}, \mathrm{c})$. Histological examination revealed SCC for both lesions (3 $\mathrm{mm}$ Breslow index for the right side lesion and $2 \mathrm{~mm}$ Breslow for the left side lesion).

\section{Discussions}

Our case, where two SCCs appear symmetrically and synchronously, is a relatively rare situation, the incidence of multiple primary cancers being reported in $0.3-4.3 \%$ cases [9].

Although the patient was retired for 20 years, the UV rays have a cumulative effect and they can cause cutaneous tumours years after exposure $[10,11]$.

The complex multidisciplinary approach of cutaneous tumours in order to optimise the surgical approach is a continuous challenge for practitioners. In our case, clinical examination pointed to a BCC diagnosis. US
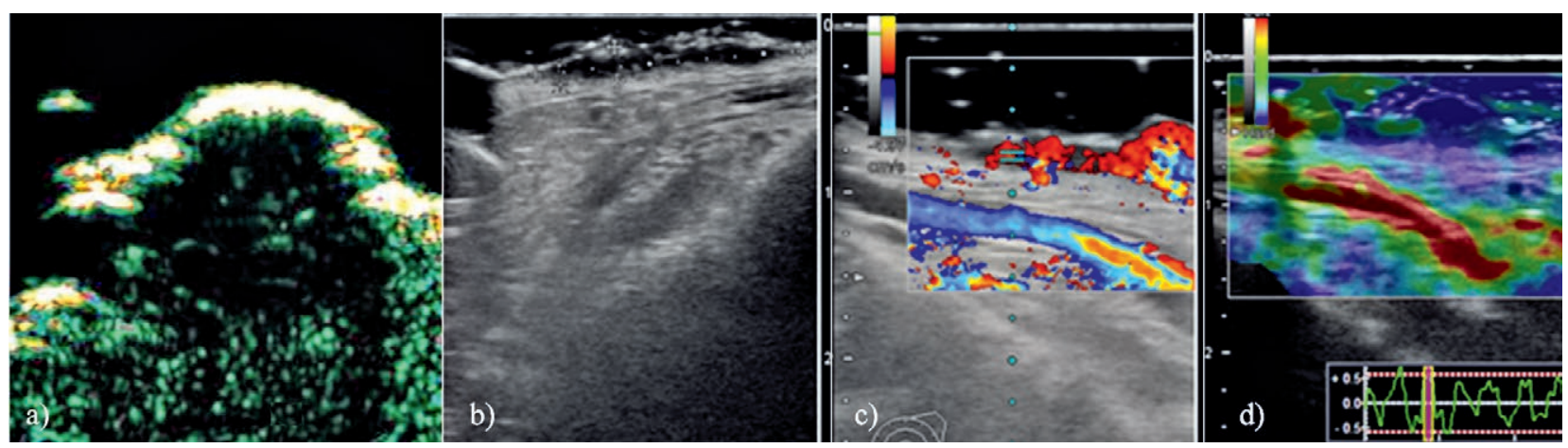

Fig 2. a) Dermascan examination: hypoechoic inhomogeneous and ulcerated tumour) located in the reticular dermis, irregular borders and sonographic depth index $3.3 \mathrm{~mm}$; the Dermascan depth value was closer to the histological Breslow index ( $3 \mathrm{~mm}$ ) compared to the conventional ultrasound depth index $(4 \mathrm{~mm})$; b) Gray scale ultrasonography: hypoecogenic ulcerated tumour located in the dermis with a depth index of $4 \mathrm{~mm}$-the right side and $3.2 \mathrm{~mm}$-the left side; c) colour Doppler examination: increased blood supply with more than 2 vascular pedicles; d) elastography showing high rigidity in the central area of the lesions, corresponding to the local hypervascularisation and tumoral infiltration. 
provided important complementary data regarding the tumour morphology, depth (quantified in $\mathrm{mm}$ as sonographic index), degree of vascularisation, and elasticity. Increased sonographic index values in comparison to the histological Breslow index values in our case can be explained by the presence of the peritumoral inflammatory infiltrate. In addition, according to the literature data and our experience, the high frequency ultrasound (Dermascan $20 \mathrm{MHz}$ ) offers highly accurate depth index information in comparison to conventional US $[8,12]$.

Intratumoral vascularisation assessed through colour Doppler revealed aspects suggesting malignant tumoural lesions. In the case of benign tumours, colour Doppler usually shows a peripheral blood supply with a slow "wash out" time while performing contrast agent investigation [6]. Qualitative elastography based on a standard colour code, revealed an increased rigidity of both tumours, suggesting malignant lesions. Inflammatory and hypervascularized lesions have increased rigidity $[6,8]$. The clinical and US data indicated the necessity of performing surgery and also gave the surgeon valuable information regarding the surgical approach.

Similar studies show the importance of US in the diagnosis and treatment of skin cancer [13,14]. This case is only one example of the importance of an interdisciplinary approach in cases with cutaneous tumours. Assessing cutaneous tumours preoperatively by means of US can lead to optimal surgical results (tumour free surgical margins and the least amount of defects).

\section{References}

1. American Cancer Society. Cancer Facts \& Figures 2017. Atlanta: American Cancer Society; 2017.

2. Kricker A, Armstrong BK, English DR, Heenan PJ. Does intermittent sun exposure cause basal cell carcinoma? A case-control study in Western Australia. Int J Cancer 1995;60:489-494.
3. Adinarayan M, Krishnamurthy SP. Clinicopathological evaluation of nonmelanoma skin cancer. Indian J Dermatol 2011;56:670-672.

4. Andrese E, Solovăstru LG, Taranu T, Iancu LS. Epidemiological and pathological aspects of skin cancer in North East of Romania. Rev Med Chir Soc Med Nat Iasi 2014;118:457-462.

5. Mourouzis C, Boynton A, Grant J, et al. Cutaneous head and neck SCCs and risk of nodal metastasis - UK experience. J Craniomaxillofac Surg 2009;37:443-447.

6. Botar-Jid CM, Cosgarea R, Bolboacă SD, et al. Assessment of Cutaneous Melanoma by Use of Very- High-Frequency Ultrasound and Real-Time Elastography. AJR Am J Roentgenol 2016;206:699-704.

7. Dasgeb B, Morris MA, Mehregan D, Siegel EL. Quantified ultrasound elastography in the assessment of cutaneous carcinoma. Br J Radiol 2015;88:20150344.

8. Crisan M, Crisan D, Sannino G, Lupsor M, Badea R, Amzica F. Ultrasonographic staging of cutaneous malignant tumors: an ultrasonographic depth index. Arch Dermatol Res 2013;305:305-313.

9. Altundag K, Yalcin S, Ozkaya O, Guler N. Synchronous squamous cell carcinoma of the stomach, the lung and the skin. Onkologie 2004;27:291-293.

10. Utrillas MP, Marín MJ, Esteve AR, et al. Ten years of measured UV Index from the Spanish UVB Radiometric Network. J Photochem Photobiol B 2013;125:1-7.

11. Ichihashi M, Ando H. The maximal cumulative solar UVB dose allowed to maintain healthy and young skin and prevent premature photoaging. Exp Dermatol 2014;23 Suppl 1:43-46.

12. Badea R, Crişan M, Lupşor M, Fodor L. Diagnosis and characterization of cutaneous tumors using combined ultrasonographic procedures (conventional and high resolution ultrasonography). Med Ultrason 2010;12:317-322.

13. MacFarlane D, Shah K, Wysong A, Wortsman X, Humphreys TR. The role of imaging in the management of patients with nonmelanoma skin cancer: Diagnostic modalities and applications. J Am Acad Dermatol 2017;76:579-588.

14. Humphreys TR, Shah K, Wysong A, Lexa F, MacFarlane $D$. The role of imaging in the management of patients with nonmelanoma skin cancer: When is imaging necessary? J Am Acad Dermatol 2017;76:591-607. 